\title{
Magnetic miniature actuators with six-DOF multimodal soft-bodied locomotion
}

\author{
Changyu $\mathrm{Xu}^{1}$, Zilin Yang${ }^{1}$, Shaun Wee Kiat Tan ${ }^{1}$, Jianhuang $\mathrm{Li}^{1}$, and Guo Zhan Lum ${ }^{1}$ \\ ${ }^{1}$ Affiliation not available
}

December 30, 2021

\begin{abstract}
Magnetic miniature robots (MMRs) are mobile actuators that can exploit their size to non-invasively access highly confined, enclosed spaces. By leveraging on such unique abilities, MMRs have great prospects to transform robotics, biomedicine and materials science. As having high dexterity is critical for MMRs to enable their targeted applications, existing MMRs have developed numerous soft-bodied gaits to locomote in various environments. However, there exist two critical limitations that have severely restricted their dexterity: (i) MMRs capable of multimodal soft-bodied locomotion have only demonstrated fivedegrees-of-freedom (five-DOF) motions because the sixth-DOF rotation about their net magnetic moment axis is uncontrollable; (ii) six-DOF MMRs have only realized one mode of soft-bodied, swimming locomotion. Here we propose a six-DOF MMR that can execute seven modes of soft-bodied locomotion and perform 3-dimensional pick-and-place operations. By optimizing its harmonic magnetization profile, our MMR can produce 1.41-63.9 folds larger sixth-DOF torque than existing MMRs with similar profiles, without compromising their traditional five-DOF actuation capabilities. The proposed MMR demonstrated unprecedented dexterity; it could jump through narrow slots to reach higher grounds; use precise orientation control to roll, two-anchor crawl and swim across tight openings with strict shape constraints; perform undulating crawling across three different planes in convoluted channels.
\end{abstract}

Keywords: Magnetic materials; soft actuators; miniature robots; locomotion.

Corresponding author(s) Email: gzlum@ntu.edu.sg

\section{Introduction}

Miniature robots are mobile actuators that are in the length scales of millimeter or smaller (Nelson et al., 2010; Hines et al., 2017; Sitti, 2018; Miskin et al., 2020). Because these actuators can leverage on their size and mobility to non-invasively access highly confined and enclosed spaces (Sitti et al., 2015; Ng et al., 2021), they are hypothesized to have the potential to revolutionize medical treatments such as minimally invasive surgery and targeted drug delivery (Li et al., 2017; Palagi et al., 2016; Jeon et al., 2019; Hu et al., 2018; Gultepe et al., 2013; Tottori et al., 2012; Felfoul et al., 2016; Esteban-Fernández et al., 2020; Alapan et al., 2020; Wu et al., 2018; Wang et al., 2021; Wang et al., 2021). These small robots have also shown to be powerful tools that can enable unprecedented lab-on-chip applications (Ceylan et al., 2017; Diller \& Sitti, 2014; Hu et al., 2021; Chung et al., 2015), and facilitate critical fundamental studies in material sciences (Tasoglu et al., 2014; Zhang et al., 2021) and biology (Palagi \& Fischer, 2018; Kim et al., 2013; Wani et al., 2017; Dong et al., 2020; Gu et al., 2020; Lum et al., 2016).

In the literature, there exist multiple methods for actuating miniature robots (Hines et al., 2017; Ilami et al., 2021). For example, these robots can be actuated via electric fields (Chen et al., 2019; Yang et al., 2015; 
Gupta et al., 2018; Liu et al., 2021), pressure (Paek et al., 2015), heat (Mourran et al., 2017), light (Palagi et al., 2016; Zeng et al., 2018; Guo et al., 2021), chemicals (Lee et al., 2010; Maeda et al., 2007), or magnetic fields (Kummer et al., 2010; Xu et al., 2021; Kim et al., 2018; Xu et al., 2019; Ceylan et al., 2021; Salehizadeh \& Diller, 2020; Kuang et al., 2021; Kim et al., 2011; Son et al., 2021). Of these methods, magnetic actuation is especially robust because the actuating magnetic fields can easily and harmlessly penetrate through most synthetic and biological materials (Kim et al., 2018; Pham et al., 2021; Sitti \& Wiersma, 2020). As a result, these magnetic miniature robots (MMRs) have great potential to operate across a diverse range of enclosed environments (Hu et al., 2018; Felfoul et al., 2016; Ren et al., 2021). In addition, unlike actuation signals such as pressure, heat and chemicals, magnetic fields can be specified not only in magnitude but also in their direction and spatial gradients (Kummer et al., 2010; Xu et al., 2021; Diller et al., 2016). By having more independent control parameters, MMRs have also shown to be the most functional among all the classes of small-scale actuators (Hu et al., 2018; Lum et al., 2016; Cui et al., 2019; Alapan et al., 2020; Zhang et al., 2021; Zhang et al., 2021; Xie et al., 2020; Pauer et al., 2021; Nguyen et al., 2021; Miyashita et al., 2017).

It is highly desirable to enhance the dexterity of MMRs because these actuators are expected to navigate across highly unstructured environments for their targeted applications (Nelson et al., 2010; Sitti, 2018; Ng et al., 2021; Ceylan et al., 2017). To enhance the agility of these actuators, the bodies of MMRs can be made soft so that they can produce a series of time-varying deformations to realize various types of locomotive gaits (Ng et al., 2021; Hu et al., 2018; Lum et al., 2016; Xu et al., 2019; Ren et al., 2021; Diller et al., 2014; Ren et al., 2019; Wang et al., 2021; Zhang \& Diller, 2015; Zhang et al., 2016; Huang et al., 2021; Ng et al., 2022). Some critical advantages of having soft-bodied locomotion are that such gaits can allow the MMRs to potentially position themselves more precisely and better adapt to various types of environments $(\mathrm{Ng}$ et al., 2021; Hu et al., 2018; Ren et al., 2021; Diller et al., 2014; Ng et al., 2022; Ze et al., 2020; Ng \& Lum, 2021; Du et al., 2020; Wu et al., 2020). There are generally two types of MMRs with soft bodies: robots that are formed by either (i) ferrofluid droplets (Fan et al., 2020; Fan et al., 2020; Liu et al., 2019; Ahmed et al., 2021) or (ii) elastomeric bodies (Hu et al., 2018; Lum et al., 2016; Xu et al., 2019; Ren et al., 2021; Ren et al., 2019). Of these two types of MMRs, those that have elastomeric bodies are especially promising. Unlike their dropletbased counterparts, the elastomer-based MMRs can produce not only dexterous locomotion but they also possess adequate stiffness necessary for performing 3-dimensional (3D) pick-and-place operations (Hu et al., 2018; Diller \& Sitti, 2014; Xu et al., 2019). In comparison to the droplet-based MMRs, the elastomer-based ones also possess higher stiffness that can allow them to transmit forces more effectively to the environment and other objects (Dong et al., 2020; Lum et al., 2016; Ren et al., 2019). By having adequate stiffness, the elastomer-based MMRs can induce sufficient impact from the substrate to produce a jumping locomotion too (Hu et al., 2018), and such complex gaits have not been demonstrated by the droplet-based soft MMRs. Due to the advantages of elastomer-based soft MMRs, we will be focusing on this class of actuators, and soft MMRs will only be referred to those that have elastomeric bodies in the subsequent text.

In recent years, the creation of soft MMRs that can generate multiple modes of locomotion represents a significant advancement for small-scale actuators (Hu et al., 2018; Ren et al., 2021; Du et al., 2020). For instance, the amphibious soft MMRs of $\mathrm{Hu}$ et al. are able to execute seven modes of soft-bodied locomotion, which can enable them to navigate across terrestrial and aquatic environments (Hu et al., 2018). Likewise, the soft MMRs of Ren et al. can use three different modes of locomotion to move in fluidic environments (Ren et al., 2021). Despite these advancements, soft MMRs with multimodal locomotive gaits are restricted to having five-degrees-of-freedom (five-DOF) motions because the sixth-DOF rotation about their net magnetic moment axis is not possible (Hu et al., 2018; Ren et al., 2021; Du et al., 2020). As a result, these MMRs can only rotate about two axes and translate along three axes (Kummer et al., 2010), and their dexterity is still far from being optimized. While soft MMRs with six-DOF do exist, such actuators have so far only realized one mode of swimming locomotion (Xu et al., 2021). This is because existing six-DOF actuators are currently limited to having a 1D magnetization profile for their soft components (Xu et al., 2021), so that their changes in geometry and magnetization profile are easy to account for during deformation. Although it is easy to implement six-DOF control on soft MMRs with 1D magnetization profile, such simple profiles have also severely constrained the dexterity of these actuators. The feasibility of executing six-DOF control 
on sophisticated MMRs, which have 2D or 3D magnetization profiles for their soft components, has not been explored yet. Due to these aforementioned limitations, the dexterity of all the existing soft MMRs is still severely restricted, and these actuators will not be able to overcome difficult barriers that have strict shape constraints. As a result, existing MMRs would not have sufficient agility to effectively negotiate across highly unstructured environments, and this poses a critical limitation for their targeted applications. In view of these challenges, it is therefore highly desirable to create dexterous MMRs that can concurrently possess six-DOF and perform multiple modes of soft-bodied locomotion.

Here we propose a six-DOF, soft MMR that can roll, two-anchor and undulating crawl, jump, swim with two different gaits, and climb a meniscus. Because the proposed MMR has six-DOF, its orientation can be precisely controlled when it executes these soft-bodied locomotive gaits, thereby allowing it to achieve very high dexterity. As the proposed six-DOF MMR can perform seven modes of soft-bodied locomotion, its dexterity will also be significantly higher than existing six-DOF soft MMRs that can only execute one type of swimming gait (Xu et al., 2021). Our proposed actuator is made up of a central magnetic beam with two identical buoyant components attached to its free ends (Fig. 1A(i), and please see supplementary information (SI) section S1 for the fabrication process and material properties of the MMR). The buoyant components are used to make the proposed soft MMR more neutrally buoyant so that it can easily swim against gravity in aquatic environments. To investigate the feasibility of implementing six-DOF control for a soft MMR with 2D magnetization profile, a single-wavelength, harmonic magnetization profile $(\vec{M})$ is programmed into our compliant beam component (Fig. 1A(ii)). We have selected a harmonic magnetization profile because this type of profile has proven to be effective for enabling multimodal locomotion (Hu et al., 2018; Ren et al., 2021).To maximize the producible sixth-DOF torque of our soft MMR, the phase shift angle of its magnetization profile, $\varphi$, has been optimized to $-90^{\circ}$ (Fig. 1A(ii) and SI section S3). With the selected $\varphi$, our proposed MMR can theoretically produce 1.41-63.9 folds larger sixth-DOF torque than existing soft MMRs that have a harmonic magnetization profile, i.e., those with $\varphi$ of $0^{\circ}$ (Zhang \& Diller, 2015; Zhang et al., 2016; Huang et al., 2021; Zhang \& Diller, 2018) and 45 (Hu et al., 2018; Ren et al., 2021; Demir et al., 2021; Culha et al., 2020) (SI section S3). Because the magnitude of our MMR's achievable net magnetic moment, $\vec{m}$, is equal to all other soft MMRs with a harmonic magnetization profile (SI section S3), this implies that all of these MMRs will have the same actuation capabilities for their traditional five-DOF motions (Kummer et al., 2010; Xu et al., 2021). The enhancement in the sixth-DOF torque for our proposed MMR has therefore been achieved without compromising their traditional five-DOF actuation capabilities. To have a fair comparison for our actuation analysis in SI section S3, we assume that all of these MMRs have the same geometries and material properties, and the key difference between them is that they possess different values of $\varphi$ in their $\vec{M}$. We have also constrained the harmonic magnetization profile of all the MMRs to be single wavelength in our analysis (SI section S3), because this has been a critical requirement for enabling multimodal soft-bodied locomotion (Hu et al., 2018; Ren et al., 2021). Using our optimized harmonic magnetization profile, the proposed MMR can be actuated to produce its desired soft-bodied locomotion and concurrently achieve optimal six-DOF motions. Since our proposed soft MMR has adequate stiffness, it can also execute 3D pick-and-place operations. 


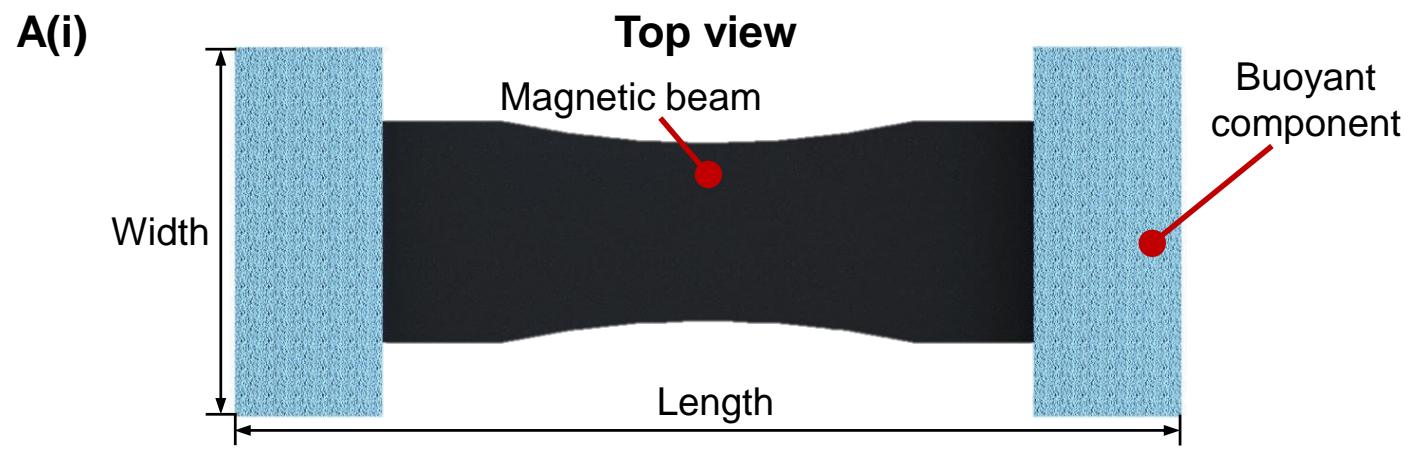

(ii)
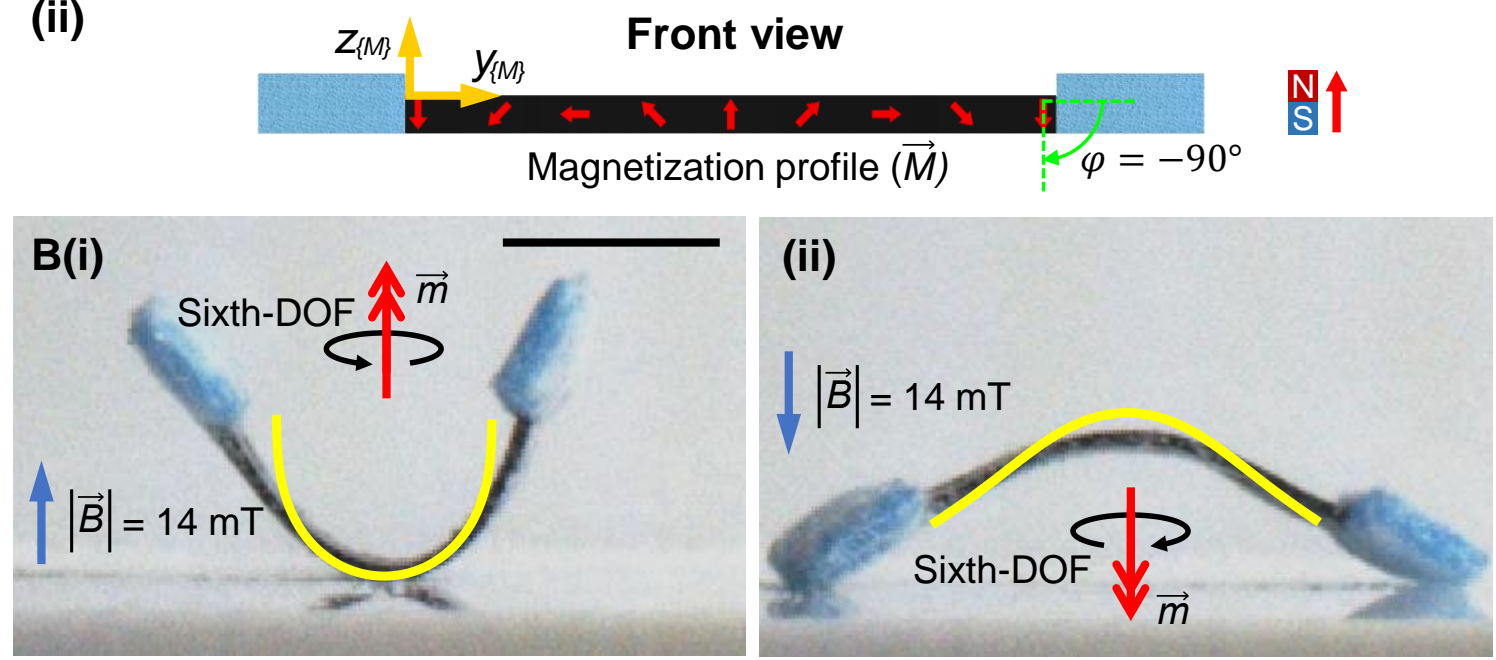


D(i)

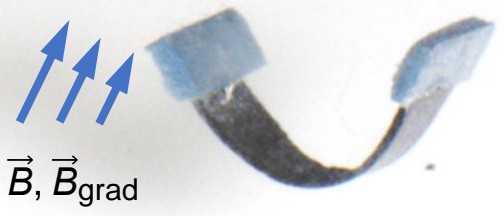

Arbitrary orientation

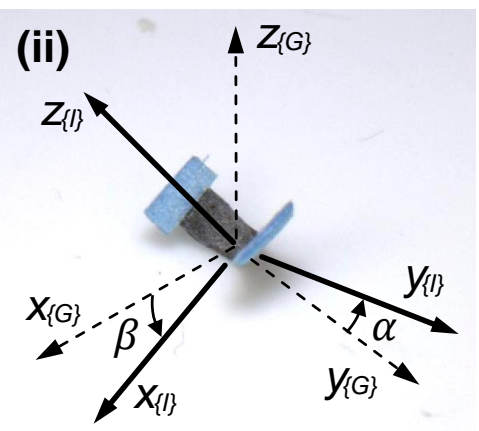

Intermediate reference frame
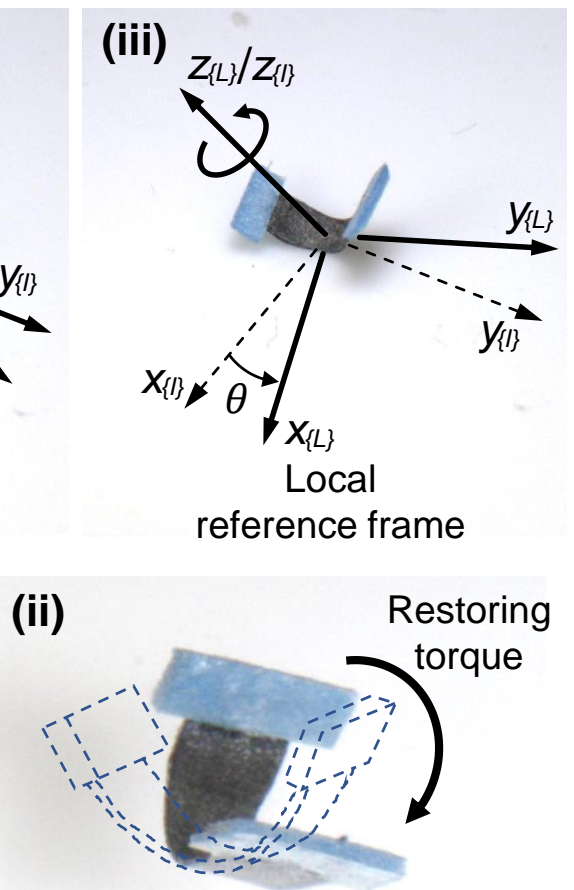

Desired orientation

Figure 1: The proposed six-DOF soft MMR. (A) The configuration of the MMR: (i) Based on the top view, the proposed MMR is made up of a central magnetic beam with two identical buoyant components attached to its free ends. (ii) The front view of the MMR that illustrates the harmonic magnetization profile of the beam with a phase shift angle $(\varphi)$ of $-90^{\circ}$. A material reference frame is attached to the MMR and its axes are denoted by the subscript $\{M\}$. (B) The two types of deformed configurations of the soft MMR: (i) The MMR assumes a ' $U$ '-shaped configuration when the magnetic field $(\vec{B})$ is applied along the positive $z_{\{M\}}$-axis. The deformed MMR will produce a corresponding net magnetic moment $(\vec{m})$ that is parallel to $\vec{B}$. (ii) The MMR 


\section{Actuation Principles}

To describe the physics of our proposed soft MMR, we first attach a material reference frame to this actuator (Fig. 1A(ii)). The axes and vectors expressed in this reference frame will be denoted by a subscript $\{M\}$. When the MMR is undeformed, its $\vec{m}$ will be a null vector (SI section S2B). However, when a magnetic field, $\vec{B}$, is applied along the positive $z_{\{M\}}$-axis, the MMR will deform and assume a ' $U$ '-shaped configuration (Fig. 1B(i) and please see SI section S2A for the physics that describes this deformation). In this deformed configuration, the MMR will possess an effective $\vec{m}$ that is parallel to the applied $\vec{B}$. Conversely, the MMR will assume an inverted 'V'-shaped configuration if the direction of $\vec{B}$ is reversed (Fig. 1B(ii)). In the inverted 'V'shaped configuration, the $\vec{m}$ of the soft MMR will be along the negative $z_{\{M\}}$-axis, parallel to the applied $\vec{B}$ (Fig. 1B(ii)). By adjusting the magnitude of $\vec{B}$, we can also actively control the curvatures of the MMR in these deformed configurations. Specifically, the curvatures will become sharper and gentler when the magnitude of $\vec{B}$ is increased and decreased, respectively (SI section S2A). In general, our theoretical model of the MMR's deformation characteristics in SI section S2A agrees with the experimental data (e.g., Fig. 1B), and this enables us to control the geometries of the MMR well. Having precise control over the curvatures of the MMR will be a critical requirement for the actuator to enable its desired modes of soft-bodied locomotion.

To analyze the proposed MMR's achievable motions in its deformed configurations, a spatial reference frame is attached to its body (Fig. 1C(iii)). This reference frame represents the local reference frame of the actuator, and the axes and vectors expressed in this frame are denoted by a subscript $\{L\}$. Because it will be easier to analyze the proposed MMR if its sixth-DOF axis is parallel to one of the principal axes in the local reference

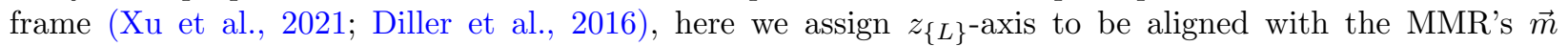
in its 'U'-shaped configuration (Fig. 1C(iii)). Based on our local reference frame assignment, the MMR's rotation about the $z_{\{L\}}$-axis is therefore its sixth-DOF motion (Xu et al., 2021; Diller et al., 2016). This frame assignment also indicates that the MMR's $\vec{m}$ will be along the negative $z_{\{L\}}$-axis when the actuator deforms to its inverted ' $V$ '-shaped configuration. To fully describe the orientation of the proposed MMR, we also introduce an intermediate reference frame and a global reference frame (Fig. 1C(i)-(ii)), and the axes and vectors expressed in these frames are denoted by the subscripts $\{I\}$ and $\{G\}$, respectively. The transformation between the global and intermediate reference frames can be described mathematically as:

$$
\vec{v}_{\{G\}}=\mathbf{R}_{x}(\alpha) \mathbf{R}_{y}(\beta) \vec{v}_{\{I\}},(1)
$$

where $\mathbf{R}_{x}$ and $\mathbf{R}_{y}$ represent the standard $x$-axis and $y$-axis rotational matrices, respectively (SI section $\mathrm{S} 2 \mathrm{~B})$. The variables, $\alpha$ and $\beta$, represent the corresponding desired angular displacements about these axes (Fig. $1 \mathrm{C}(\mathrm{i})$-(ii)), while the vector, $\vec{v}$, represents an arbitrary vector. It is noteworthy that the $z_{\{I\}}$-axis of the MMR defines the desired orientation of its $\vec{m}$, implying that the direction of the sixth-DOF axis can be fully defined based on the values of $\alpha$ and $\beta$. In a similar way, the transformation between the intermediate and local reference frames can be described mathematically as:

$$
\vec{v}_{\{I\}}=\mathbf{R}_{z}(\theta) \vec{v}_{\{L\}},(2)
$$

where $\mathbf{R}_{z}$ represents the standard $z$-axis rotational matrix (SI section S2B) and $\theta$ represents the desired sixth-DOF angular displacement of the MMR (Fig. 1C(ii)-(iii)).

To enable precise orientation control for the proposed MMR, we control $\vec{B}$ and its independent spatial gradients, $\vec{B}_{\text {grad }}$, in such a way that the desired orientation of the MMR becomes a minimum potential energy configuration (SI section S2B-C). Under the influence of these actuating magnetic signals, the deformed MMR will constantly experience three axes of restoring torques until it self-aligns to the desired orientation (Fig. 1D 
and SI section S2B). The presence of the restoring torques will also help the proposed MMR to reject external disturbances, enabling this actuator to maintain its desired orientation at all times (SI section S2B). Of the three axes of restoring torques, two of them are induced by the phenomenon in which the MMR's $\vec{m}$ will tend to align with the applied $\vec{B}$ (Tasoglu et al., 2014; Lum et al., 2016; Kummer et al., 2010). By exploiting this phenomenon, we can therefore control the MMR's two axes of angular displacements, $\alpha$ and $\beta$, via specifying the direction of $\vec{B}$ (SI section S2B). However, controlling the direction of $\vec{B}$ will not allow us to exert the third restoring torque on the MMR, i.e., the restoring torque about its sixth-DOF axis (Kummer et al., 2010; Xu et al., 2021; Diller et al., 2016; Diller et al., 2014). The sixth-DOF restoring torque can instead be induced on the MMR via controlling $\vec{B}_{\text {grad }}$, and this torque will enable us to precisely control $\theta$ (SI section S2B-C). By controlling $\vec{B}_{\text {grad }}$, we can also apply desired magnetic forces on the MMR and allow it to translate along three axes so as to achieve another three-DOF (SI section S2B). A notable advantage of our control scheme is that we can fully decouple all the magnetic torques and forces applied to the MMR, effectively allowing the soft actuator to achieve six-DOF motions (SI section S2B-C). Together with controlling the time-varying deformations of the MMR, such six-DOF control will be critical for empowering our proposed actuator to execute multiple modes of soft-bodied locomotion with precise orientation control. Our six-DOF control strategy can be implemented successfully because we have modelled how the critical robotic parameters of our MMR will vary as the actuator changes its geometry and $\vec{M}$ during deformation (SI sections S2B-C and S3). Examples of such robotic parameters will include but not limited to the MMR's $\vec{m}$ and producible normalized sixth-DOF torque (SI sections S2B-C and S3).

\section{Experimental Results}

We first evaluated the sixth-DOF torque, $T_{z,\{L\}}$, producible by our proposed soft MMR. Because the producible $T_{z,\{L\}}$ of the MMR would vary when this actuator underwent different amounts of deformation (SI section S3), here we constrained the MMR to its undeformed configuration for easier characterization purposes. As the buoyant components of the proposed MMR were passive and they could not produce $T_{z,\{L\}}$ for the MMR, these components were removed in this experiment. The undeformed magnetic beam component of the MMR was fixed at the free end of another fixed-free beam in this experiment (Fig. 2A). By observing how the fixed-free beam would deform as we varied the applied actuating magnetic signals to the proposed MMR, we could deduce the producible $T_{z,\{L\}}$ of our actuator. This could be achieved by using a camera to record the angular deflection of the fixed-free beam's free end, $\theta_{\text {tip }}$, and subsequently applying the Euler-Bernoulli equation to deduce the generated $T_{z,\{L\}}$ (Fig. 2A and SI section S4B). Based on this methodology, we established the relationship between the MMR's generated $T_{z,\{L\}}$ and the applied magnetic signals (Fig. 2B). Each data point in the graph of Fig. 2B had five trials, and the gradient of the best fit line represented the MMR's producible $T_{z,\{L\}}$ after it was normalized according to the strength of the actuating signals. The normalized sixth-DOF torque of our MMR was experimentally evaluated to be $1.16 \times 10^{-7} \mathrm{~N} \mathrm{~m}^{2}$ $\mathrm{T}^{-1}$, and there was a $13.7 \%$ deviation from our simulation predictions in SI section S3 (predicted value: $1.02 \times 10^{-7} \mathrm{~N} \mathrm{~m}^{2} \mathrm{~T}^{-1}$ ). In general, the experimental data agreed with the prediction from our simulations, and the deviation could be caused by minor fabrication errors and parallax measurement errors from the camera. Another potential source of experimental error was that the MMR may not remain perfectly in its undeformed configuration during the experiments, and this might cause the evaluated normalized sixth-DOF torque to be larger than expected (SI section S3). As our theoretical model could also predict the MMR's deformation characteristics well (Fig. 1B and SI section S2A), the data in Fig. 2B supported our hypothesis that the proposed MMR could indeed produce 1.41-63.9 folds larger $T_{z,\{L\}}$ than existing soft MMRs with single-wavelength, harmonic magnetization profiles (Hu et al., 2018; Ren et al., 2021; Diller et al., 2014; Zhang \& Diller, 2015; Zhang et al., 2016; Zhang \& Diller, 2018; Demir et al., 2021; Culha et al., 2020) (SI section S3). After investigating the producible sixth-DOF torque of our proposed MMR, we proceeded to evaluate the locomotion of this actuator. 
Figure 2: An experiment that investigated the sixth-DOF torque producible by the proposed MMR. (A) The magnetic beam component of the MMR was fixed at the free end of a larger fixed-free beam. As the sixth-DOF torque of the magnetic beam $\left(T_{z,\{L\}}\right)$ was generated, the fixed-free beam's angular deflection at the free end, $\theta_{\text {tip }}$, would be recorded by a camera. In this experiment, the magnetic beam was constrained to be in its undeformed configuration. (B) The experimental plot of the deduced $T_{z,\{L\}}$ against the actuating magnetic signals $\left(\frac{\partial B_{x,\{L\}}}{\partial y\{L\}}\right)$. Five trials were conducted for each data point and the error bars represented the corresponding standard deviation of the data points. The gradient of the best fit line was computed to be $1.16 \times 10^{-7} \mathrm{~N} \mathrm{~m}^{2} \mathrm{~T}^{-1}$, and it represented the normalized sixth-DOF torque of the proposed MMR (in its undeformed configuration). Scale bar: $2 \mathrm{~mm}$.

Rolling could be considered as one of the fastest modes of terrestrial locomotion ( $\mathrm{Ng}$ et al., 2021; Hu et al., 2018). Our proposed MMR could execute this locomotion by first applying a $\vec{B}$ of $13 \mathrm{mT}$ along its positive $z_{\{L\}}$-axis so that it could assume a ' $U$ '-shaped configuration (Fig. $3 \mathrm{~A}$ ). It is advantageous to adopt such a configuration since this would be a favorable shape for the MMR to roll (Hu et al., 2018). Subsequently, the MMR would be able to start rolling when we continuously rotate the magnetic field in the $y_{\{L\}} z_{\{L\}}$ or $z_{\{L\}} x_{\{L\}}$ planes $(|\vec{B}|=13 \mathrm{mT})$. Because our proposed MMR had six-DOF, this actuator could choose to roll along its length (rotating about the $x_{\{L\}}$-axis) or along its width (rotating about the $y_{\{L\}}$-axis) (Fig. $3 \mathrm{~A}$ and SI Video S1). The rolling direction of the MMR could also be steered via controlling its angular displacement about the $z_{\{L\}}$-axis with $\vec{B}_{\text {grad }}$ (Fig. $3 \mathrm{~B}$ and SI Video S1). In comparison, rolling along the length of the MMR would be faster than rolling along its width since the length of our actuator was longer than its width (Fig. 1A and SI section S4C). This is assuming that the rotating frequencies of $\vec{B}$ were identical for both rolling scenarios. Despite being slower, rolling along the width of the MMR had its unique merits too. Because the curvatures of the actuator could be controlled to be much gentler for this mode of rolling (Fig. S11), the MMR could potentially squeeze through smaller openings if it was rolling along its width. By having two modes of rolling, the proposed MMR was therefore very robust. On obstacle-free terrains, it could choose to roll quickly along its length $\left(2.46 \mathrm{~mm} \mathrm{~s}^{-1}\right.$ with a $0.25 \mathrm{~Hz}$ rotating $\vec{B}$ ). Alternatively, it could choose to roll along its width if it had to negotiate across barriers with narrow openings in the environment $\left(1.85 \mathrm{~mm} \mathrm{~s}^{-1}\right.$ with a $0.25 \mathrm{~Hz}$ rotating $\vec{B}$ ). While existing rolling MMRs were also steerable, they had only demonstrated rolling along their lengths (Hu et al., 2018; Gu et al., 2020; Ren et al., 2021; Yang et al., 2020). As a result, our proposed MMR would be much more dexterous than existing rolling MMRs (Hu et al., 2018; Gu et al., 2020; Ren et al., 2021; Yang et al., 2020) because it would have greater versatility to roll across environments that have difficult barriers. To demonstrate the remaining DOF of our proposed MMR, we commanded it to translate along its three principal axes in the local reference frame (Fig. 3C and SI Video S1). Before executing these translations, we first applied a $\vec{B}$ along the $z_{\{L\}}$-axis of the MMR so that it could deform into a 'U'-shaped configuration to possess an effective $\vec{m}$. Corresponding $\vec{B}_{\text {grad }}$ was then applied to exert the desired magnetic forces on the MMR to translate it along the $x_{\{L\}^{-}}, y_{\{L\}^{-}}$, and $z_{\{L\}^{-}}$axes (Fig. 3C and SI Video S1). During the translations along the $x_{\{L\}^{-}}$and $y_{\{L\}^{-a x e s}}$, an upward magnetic force was also applied to the MMR so that it could reduce the normal force and thus friction induced by the substrate (Fig. 3C(i)-(ii)). A notable observation in the experiments shown in Fig. 3A-C and SI Video S1 was that the rotations and translations of the proposed MMR could be fully decoupled, implying that this actuator was able to attain six-DOF control. It would be advantageous to be able to apply magnetic forces on the proposed MMR because these forces could potentially enhance the efficiency of the actuator's locomotion (Hu et al., 2018). For instance, we could apply upward forces on the MMR so that it could reduce its friction with the ground, jump higher or swim against gravity easier. However, translating the MMR solely by magnetic forces might be detrimental because it would be challenging to position the actuator precisely with such control strategies (Hu et al., 2018; Zhang et al., 2015). 

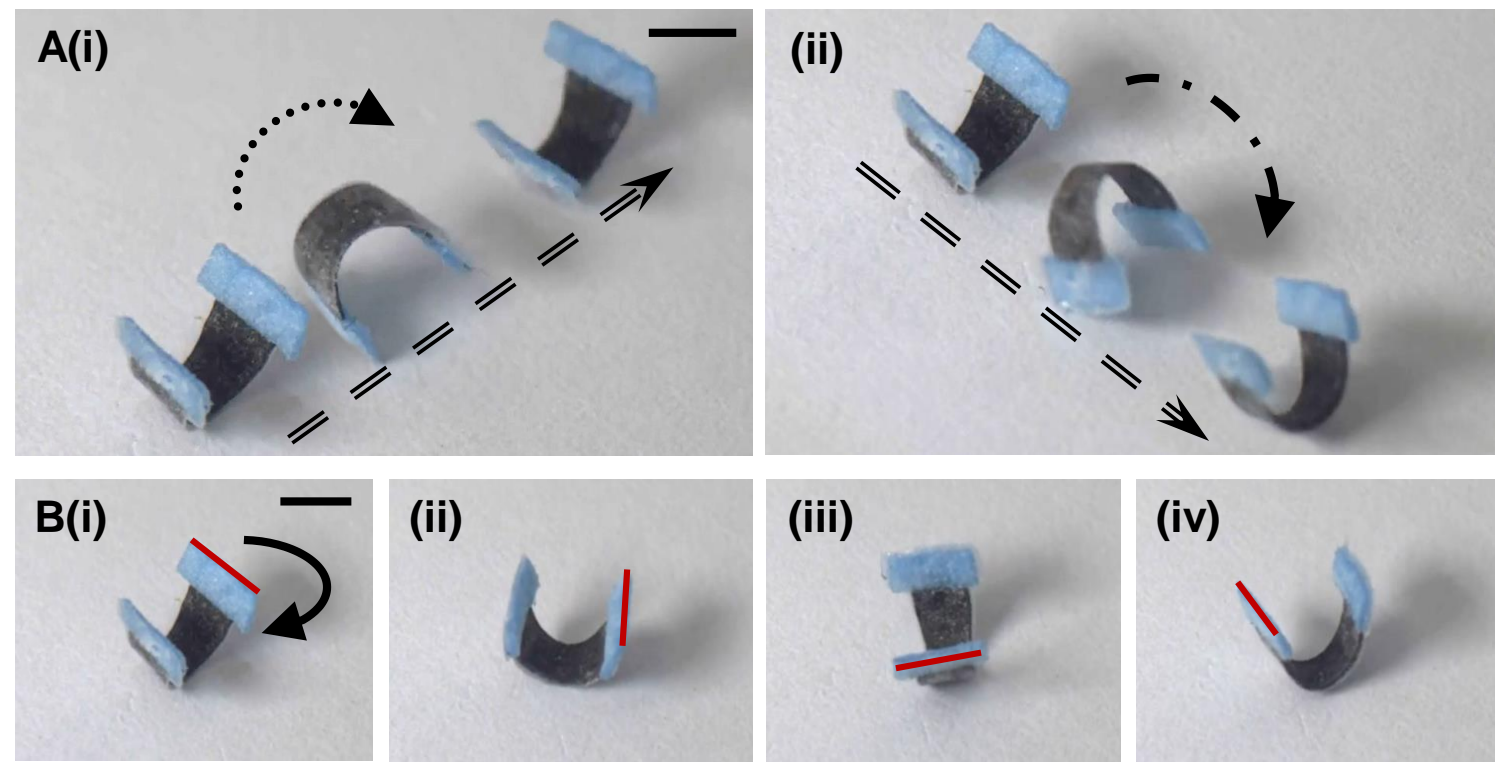

(ii)

(iii)
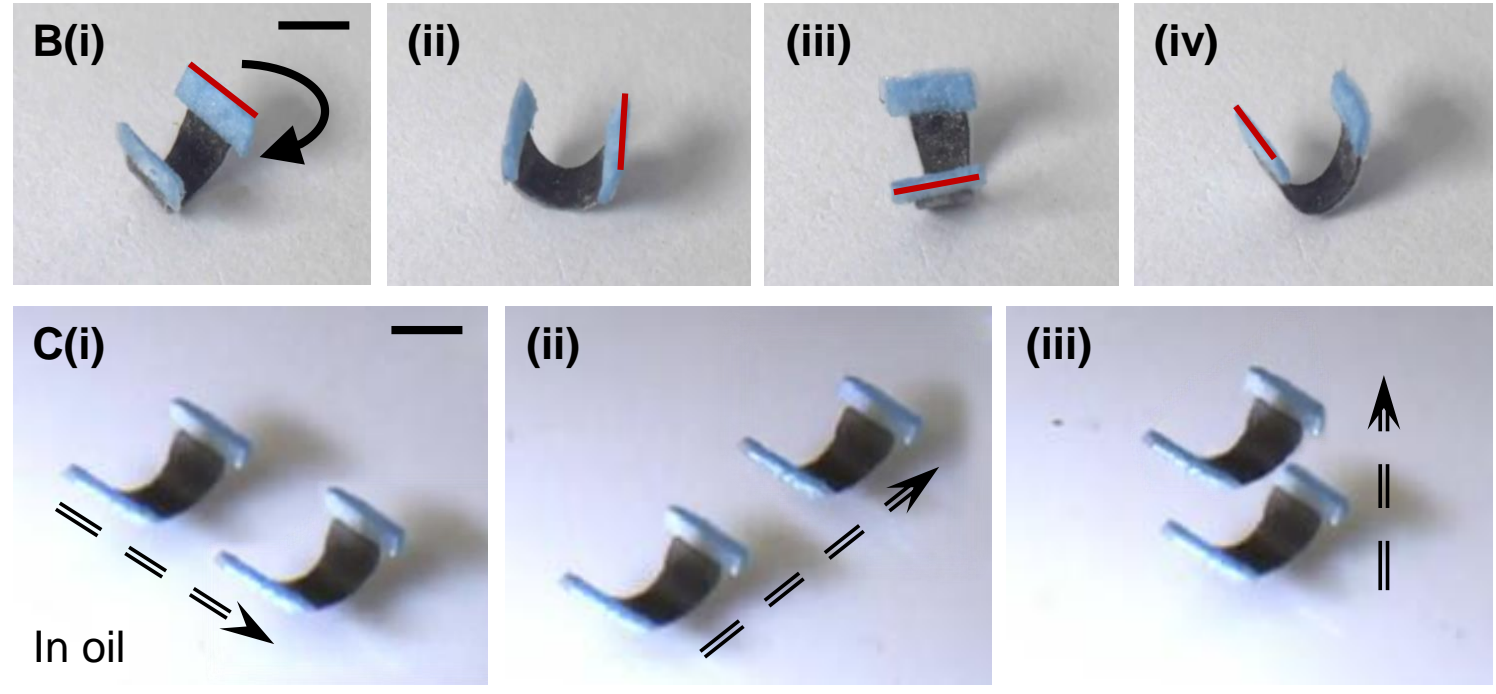

(iii)
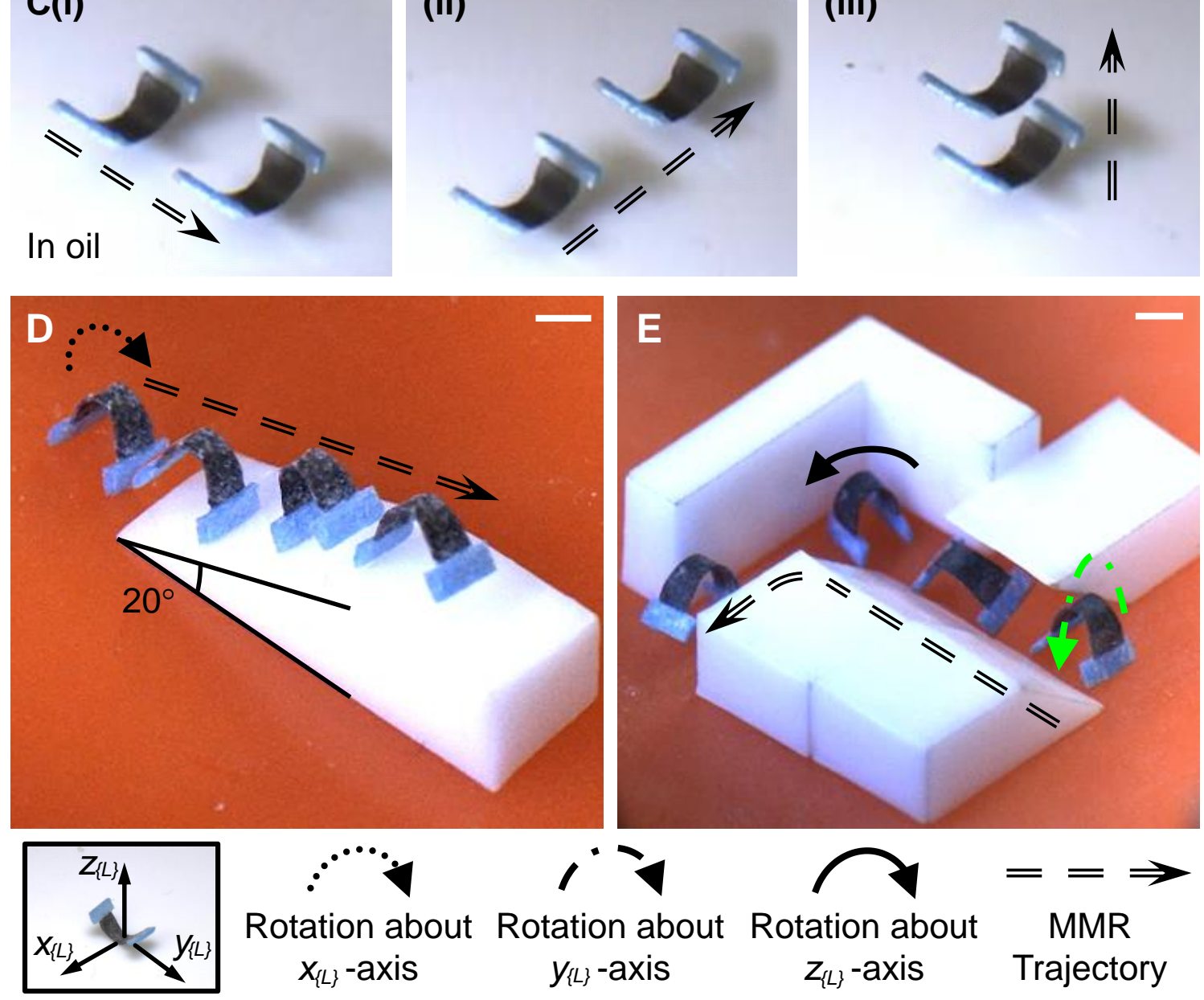

Figure 3: The rolling, translations, and two-anchor crawling locomotion of the proposed MMR. Except for (B), the remaining sub-figures were created by superp8sitioning the snapshots of the MMR at different timestamps in SI Videos S1-2. (A) The rolling locomotion of the MMR: (i) Rolling along the length of the MMR by continuously rotating it about the $x_{\{L\}}$-axis. (ii) Rolling along the width of the MMR by continuously rotating it about the $y_{\{L\}}$-axis. This mode of rolling could be accomplished with gentler curvatures compared to rolling along the MMR's length. (B) The MMR's sixth-DOF rotation (rotating about the $z_{\{L\}}$-axis). This rotation enabled the MMR to steer its rolling locomotion. A red line was added to one of the MMR's free ends to better illustrate this rotation. (C) Translating the MMR in a naraffin oil reservoir. The MMR was 


\section{SI Video S1}

\section{Rolling locomotion and translations}

Rich media available at https://drive.google.com/file/d/1Fpdxa0B0psp6XWmRz3YF9MhgvVChZS1X/ view?usp=sharing

In addition to rolling and translations, our proposed MMR could also execute two-anchor crawling locomotion. A significant advantage of this terrestrial locomotion was that it could allow the MMR to position itself more precisely in unstructured environments compared to rolling (Hu et al., 2018). This is because this mode of locomotion allowed the MMR to precisely tune its stride length (Hu et al., 2018). To implement the two-anchor crawling locomotion, we used the free ends of the MMR to serve as the two contact points with the substrate. This locomotion was cyclic, and each cycle began by first allowing the MMR to assume a 'U'-shaped configuration and allowing both of its free ends to be in contact with the substrate (Fig. S12D(i),

the applied $\vec{B}$ was along $z_{\{L\}}$-axis with a magnitude of $18 \mathrm{mT}$ ). Subsequently, we would anchor the MMR with its rear end so that it could lift the front end of the body upwards via a rotation about the $x_{\{L\}}$-axis (Fig. S12D(ii)). This could be achieved by rotating the applied $\vec{B}$ counterclockwise $\left(15-30^{\circ}\right)$ in the $y_{\{L\}} z_{\{L\}}$ plane. Once the front end of the MMR was lifted, we rotated $\vec{B}$ clockwise back to its original direction and reduced its magnitude to $4 \mathrm{mT}$ so that the actuator could extend and produce a net displacement forward (Fig. S12D(iii)). Following this extension, the front end of the MMR would resume contact with the substrate and serve as the new anchor point. By rotating $\vec{B}$ clockwise in the $y_{\{L\}} z_{\{L\}}$ plane $\left(15-30^{\circ}\right)$ while increasing its magnitude to $18 \mathrm{mT}$, we could lift and contract the rear end of the MMR (Fig. S12D(iv)). Thereafter, we rotated $\vec{B}$ counterclockwise back to its original direction to complete one cycle and produce a net displacement (Fig. S12D(v)). Since the proposed MMR possessed six-DOF, we could control this actuator's sixth-DOF angular displacement about its $z_{\{L\}}$-axis to steer it along the desired direction. In addition, we could also rotate the MMR about its $y_{\{L\}}$-axis and tilt its body at an angle as it executed the two-anchor crawling locomotion. By tilting the MMR, this actuator could better conform to the strict shape constraints of a narrow passage so that it could easily cross this passage with the two-anchor crawling locomotion (Fig. $3 \mathrm{E}$ and SI Video S2). With six-DOF control, the MMR could also control the angular displacements about its $x_{\{L\}}$-axis and perform the two-anchor crawling locomotion to ascend an inclined slope of $20^{\circ}$ (Fig. 3D and SI Video S2), and successfully execute sharp turns by rotating about its $z_{\{L\}}$-axis at the junction of a confined 'L'-shaped path (Fig. 3E and SI Video S2). Although existing MMRs were steerable when they executed the two-anchor crawling locomotion on obstacle-free flat terrains (Hu et al., 2018; Demir et al., 2021; Culha et al., 2020), they could not use this locomotion to concurrently accomplish the following tasks: (i) climb an inclined slope; (ii) perform effective turns on sharp corners; and (iii) tilt at an angle to better conform and thus crossing a narrow passage with strict shape constraints. The proposed MMR therefore demonstrated significantly higher dexterity than existing MMRs that could perform two-anchor crawling locomotion (Hu et al., 2018; Kim et al., 2011; Wu et al., 2020; Demir et al., 2021; Culha et al., 2020; Yang et al., 2020; Ju et al., 2021).

\section{SI Video S2}

\section{Two-anchor crawling locomotion}

Rich media available at https://drive.google.com/file/d/15zbajXe3WNx11Ra0QKLjivvbiayIMF5o/ view?usp=sharing 
Terrestrial locomotion like rolling and two-anchor crawling might not be applicable if the MMRs had to negotiate across highly confined and enclosed channels. In such scenarios, executing undulating crawling would be an effective locomotion for MMRs to overcome such obstacles (Hu et al., 2018; Ren et al., 2021). When our proposed MMR entered such channels, we could continuously rotate $\vec{B}(15-20 \mathrm{mT})$ in the $y_{\{L\}} z_{\{L\}}$ plane so that the actuator could undulate and generate a traveling wave along its body (Fig. 4 and SI Video S3). Due to the generated traveling wave, the proposed MMR was able to induce a net propulsive force from the surroundings to thrust itself forward. Since our MMR had six-DOF, it could precisely control the angular displacements about its $y_{\{L\}}$ - and $z_{\{L\}}$-axes to negotiate through a complex channel that required it to sequentially undulating crawl across two perpendicular planes (Fig. 4A and SI Video S3). The MMR could also execute undulating crawling to ascend an inclined slope of $20^{\circ}$ by controlling the angular displacement about its $x_{\{L\}}$-axis, and rotate about its $z_{\{L\}}$-axis to make a sharp turn at the junction of a confined ' $\mathrm{L}$ 'shaped channel (Fig. 4B and SI Video S3). As previous MMRs could only demonstrate undulating crawling through straight or planar channels (Hu et al., 2018; Ren et al., 2021; Du et al., 2020), the advancement of our proposed actuator was therefore highly significant since it could use this mode of locomotion to negotiate across a much more diverse range of confined, enclosed channels that had convoluted 3D geometries. 

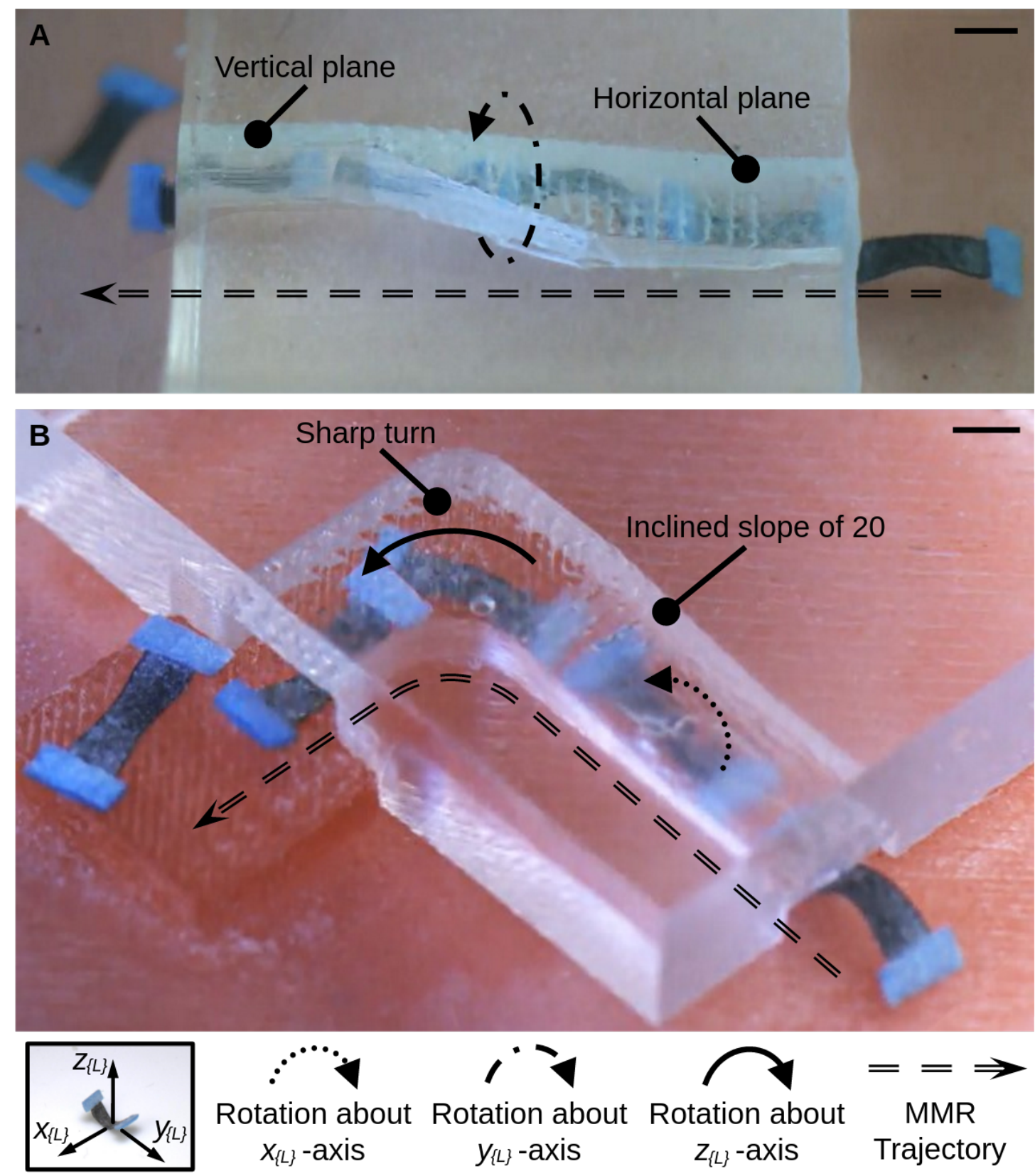

Figure 4: A demonstration of the MMR's undulating crawling locomotion. The sub-figures were created by superpositioning the snapshots of the MMR at different timestamps in SI Video S3. (A) The MMR could use its undulating crawling locomotion to negotiate through a complex channel by using this gait to crawl across two perpendicular planes. During this process, the MMR had to control its angular displacement about the $y_{\{L\}}$-axis to transit between the planes. (B) The MMR could execute undulating crawling to ascend an inclined slope of $20^{\circ}$ by controlling its angular displacements about the $x_{\{L\}}$-axis. By rotating about its $z_{\{L\}}$-axis, the MMR could perform a sharp turn at the junction of a confined ' $L$ '-shaped channel. The legend showed the local reference frame of the MMR as well as the types of arrows representing rotations about different axes and the MMR's trajectory. Scale bars: $2 \mathrm{~mm}$. 


\section{SI Video S3}

\section{Undulating crawling locomotion}

Rich media available at https://drive.google.com/file/d/1VGC31_9Abg8G6edJb9QDcpa_pU3owgIM/ view?usp=sharing

Similar to undulating crawling, jumping would be an effective locomotion for the MMR to overcome difficult obstacles (Ng et al., 2021; Hu et al., 2018). Specifically, MMRs could execute such locomotion to jump across barriers that had heights greater than their body length (Ng et al., 2021; Hu et al., 2018) . The jumping locomotion could be executed by first deforming the proposed actuator into a 'U'-shaped configuration (the applied $\vec{B}$ was along $z_{\{L\}}$-axis with a magnitude of $20 \mathrm{mT}$ ) and then orientating the MMR's $y_{\{L\}} z_{\{L\}}$ plane such that its $z_{\{L\}}$-axis had a clockwise $5^{\circ}$ angle away from the vertical upright direction. Subsequently, we rotated $\vec{B}$ clockwise in the $z_{\{L\}} x_{\{L\}}$ plane $\left(175^{\circ}\right)$ via a step change so that a strong magnetic torque could be exerted on the MMR to enable a rigid-body rotation while the actuator concurrently underwent a deformation. During this rotation and shape-changing process, the free ends of the MMR would eventually strike the substrate and gain a momentum to jump against gravity (Fig. 5A and SI Video S4). To allow the proposed MMR to jump higher, we also applied an upward magnetic force to this actuator via $\vec{B}_{\text {grad }}$ (jumping height: $6.8 \mathrm{~mm} / 1.06$ body length, jumping range: $9.56-11.2 \mathrm{~mm} / 1.49-1.75$ body length). In general, we could


desired horizontal projectile motion before initiating the jump. A notable advantage of our proposed actuator over existing soft jumping MMRs (i.e., (Hu et al., 2018; Zhong \& Wei, 2021; Wang et al., 2021)) was that it could precisely control its three axes of angular displacements in mid-air after the jumping locomotion was executed. For example, we had shown that the proposed MMR could control its angular displacements about the $z_{\{L\}^{-}}$and $x_{\{L\}^{-a x e s}}$ while it executed the jumping locomotion in Fig. 5A(i) and SI Video S4. Similarly, this MMR could also precisely control its angular displacements about the $z_{\{L\}}$ and $y_{\{L\}}$-axes while it performed the jump in Fig. 5A(ii) and SI Video S4. By having the ability to precisely control its orientation during a jump, our proposed MMR could therefore have the potential to adjust itself into a favorable shape necessary for jumping across barriers, which have openings with strict shape constraints. Such an ability would be highly desirable, but it had not been achieved by existing jumping MMRs yet (Ng et al., 2021; Hu et al., 2018; Zhong \& Wei, 2021; Wang et al., 2021). 

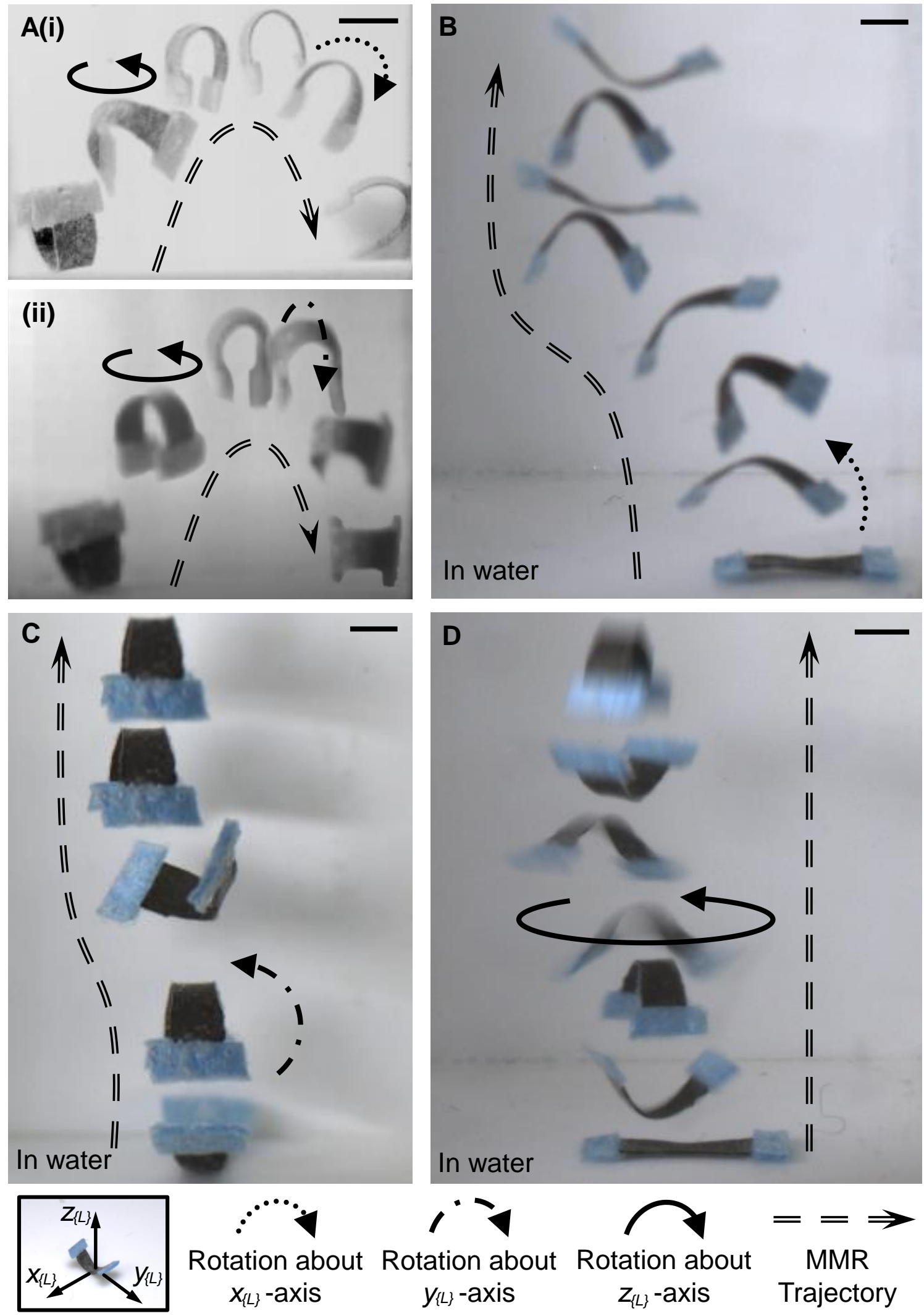

$y_{\{L\}}$-axis

Figure 5: Demonstrating the jumping and jellyfish-like swimming locomotion of the proposed MMR. The sub-figures were created by superpositioning the snapksots of the MMR at different timestamps in SI Videos S4-5. (A) The MMR's jumping locomotion: (i) During the jump, the MMR could control its angular displacements about the $z_{\{L\}}$ - and $x_{\{L\}}$-axes. (ii) During this jump, the MMR showed that it could control its angular displacements about the $z_{\{L\}^{-}}$and $y_{\{L\}}$-axes. (B-D) The jellyfish-like swimming locomotion of the MMR. (B) As the MMR swam, it could control its angular displacement about the $x_{\{L\}}$-axis to steer the swimming direction. (C) Similar to (B), the MMR could steer the swimming direction by controlling its anoular displacement about the $u$ - -axis. (D) The MMR could also continuously rotate about its sixth-DOF 


\section{SI Video S4}

\section{Jumping locomotion}

Rich media available at https://drive.google.com/file/d/1btoz2Q53bhX530tsKiGaSlpl8LxhZ7ai/ view?usp=sharing

As an amphibious robot, our proposed MMR could also mimic the swimming locomotion of a biological jellyfish to maneuver in water bulk. This soft locomotive gait could be realized by oscillating $\vec{B}$ along the $z_{\{L\}^{-}}$ axis of the MMR so that the actuator could alternate between its ' $U$ '- and inverted ' $V$ '-shaped configurations (SI Video S5). By oscillating $\vec{B}$ at a frequency of $6-8 \mathrm{~Hz}$ (maximum $|\vec{B}|: 24 \mathrm{mT}$ ), the proposed MMR could induce a net propulsive force from its surroundings to swim against gravity (average velocity: 7.65-10.4 mm $\mathrm{s}^{-1}$ ). It was possible to swim with this reciprocal gait because the MMR was able to attain an adequately high Reynolds number of 54.9-74.6 to gain sufficient inertia effects (SI Section S4D). The swimming direction of the MMR could also be steered by controlling its angular displacements about the $x_{\{L\}^{-}}$and $y_{\{L\}}$-axes to dictate the propulsion direction (Fig. 5B-C and SI Video S5). As it swam, the proposed MMR could also rotate about its sixth-DOF axis (the $z_{\{L\}}$-axis) via controlling $\vec{B}_{\text {grad }}$ (Fig. $5 \mathrm{D}$ and SI Video S5). Because we could precisely control the MMR's three axes of angular displacements, we could potentially command this actuator to adjust its orientation and adopt a favorable shape to easily swim through barriers with openings that had strict shape constraints. Having such dexterity would be a significant advancement over existing five-DOF MMRs that could perform jellyfish-like swimming locomotion (Hu et al., 2018; Lum et al., 2016; Ren et al., 2019). This is because although existing five-DOF, jellyfish-like MMRs could also steer their swimming direction, they would lack the required sixth-DOF dexterity to swim across complicated barriers (Hu et al., 2018; Lum et al., 2016; Ren et al., 2019). While the six-DOF soft MMR in our previous work could also fully control its orientation to swim across difficult barriers, it was restricted to having one soft-bodied locomotion (Xu et al., 2021). Thus, our previous actuator in (Xu et al., 2021) would not be able to perform the other types of gaits demonstrated by our proposed MMR.

\section{SI Video $S 5$}

\section{Jellyfish-like swimming locomotion}

Rich media available at https://drive.google.com/file/d/1jr6sykD4mAAvEuvVFFcBA7oBI_87rQ53/ view?usp=sharing

Because our proposed MMR could concurrently possess six-DOF and execute multimodal soft-bodied locomotion, it was able to demonstrate significantly higher dexterity than existing MMRs (e.g., (Hu et al., 2018; Xu et al., 2021; Ren et al., 2021; Ren et al., 2019; Du et al., 2020)). To demonstrate such capabilities, we commanded our MMR to negotiate across three synthetic, unstructured environments that had challenging obstacles. For the first unstructured environment, the MMR began by jumping through a narrow slot to reach a higher ground (height: $6.5 \mathrm{~mm}$ ) (Fig. 6A and SI Video S6). This was possible because the MMR was able to adjust its orientation during the jump such that it could adopt a favorable shape to fit through the slot. An additional video was taken in slow motion to better illustrate this jumping process (Fig. 6B and SI Video S6). Once the actuator arrived at the higher ground, it could use its two-anchor crawling locomotion to precisely maneuver itself to reach the narrow opening of a wall on its right (Fig. 6A and SI Video S6). In order to bypass this wall, the MMR had to roll along its width (rotating about the $y_{\{L\}}$-axis) so that it could adopt a gentle curvature necessary for squeezing through the wall's narrow opening (Fig. 6A and SI 
Video S6, average speed: $0.37 \mathrm{~mm} \mathrm{~s}^{-1}$ ); otherwise, it would not be able to roll across this rigid barrier. After passing through this wall, the MMR controlled its sixth-DOF angular displacement about the $z_{\{L\}}$-axis to steer the rolling direction through the opening of the final wall. Because this opening was much larger than the previous one, the MMR could pass through by rolling along its length (rotating about the $x_{\{L\}}$-axis) and achieved a relatively fast rolling speed to reach the final destination (SI Video S6, average speed: 0.46 $\mathrm{mm} \mathrm{s}^{-1}$ ). It is noteworthy that existing five-DOF MMRs, which could execute multimodal soft-bodied locomotion, would not be able to negotiate across this unstructured environment (Hu et al., 2018; Ren et al., 2021; Du et al., 2020). This is because such existing soft MMRs would neither be able to fully control their orientation to jump through narrow slots nor roll along their width (shorter side). 

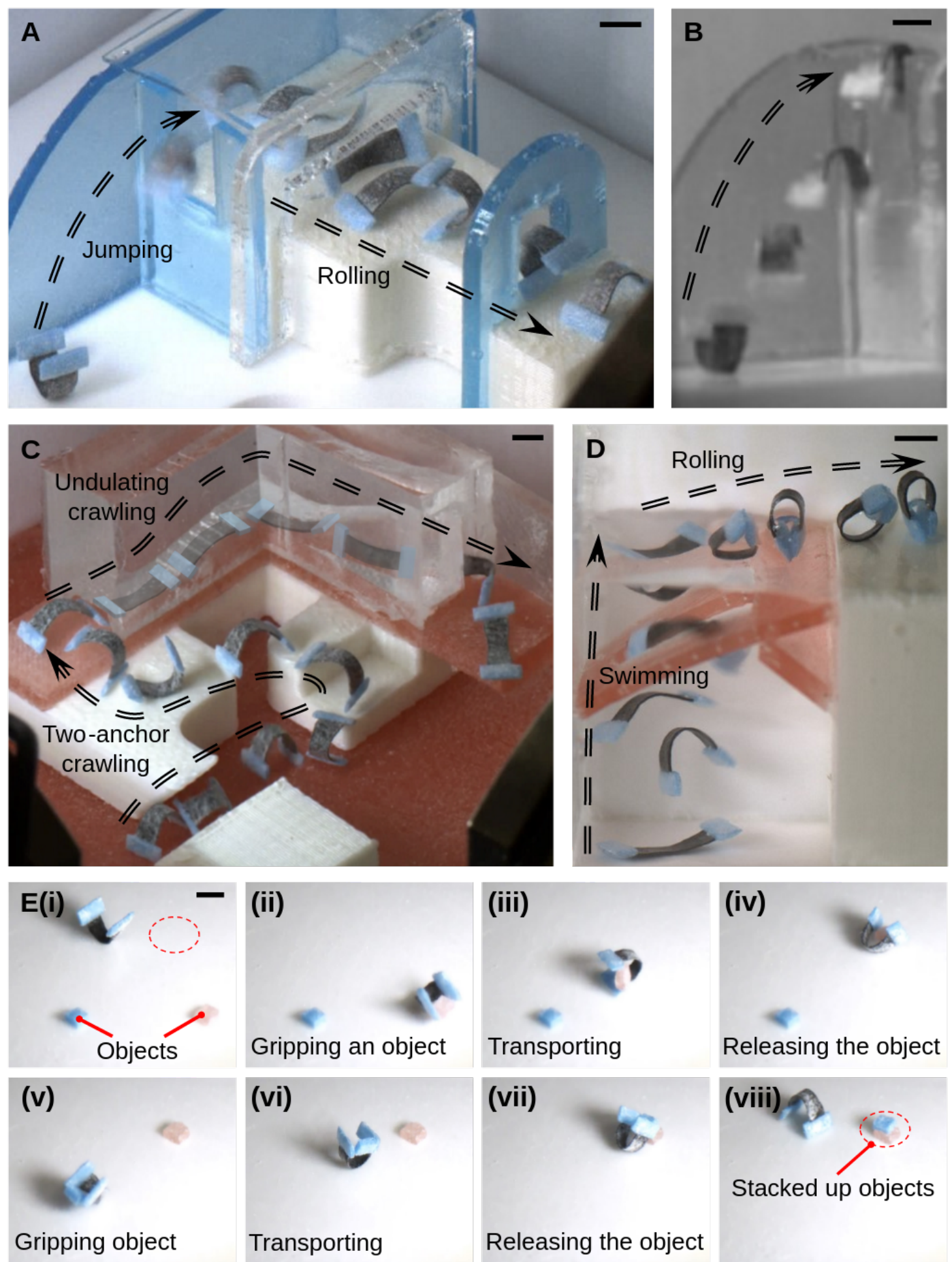

Figure 6: The six-DOF MMR utilized its multimodal soft-bodied locomotion to navigate through various synthetic, unstructured environments with complex barriers and performed a 3D pick-and-place operation. Except for (E), the sub-figures were created by superpositioning the snapshots of the MMR at different timestamps in SI Videos S6-8. (A) The MMR navigated through an unstructured terrestrial environment via the jumping and rolling locomotion. The MMR first arrived at a higher ground by jumping through a narrow slot via precisely controlling its orientation. Subsequently, the MMR rolled along its width with a gentle curvature to squeeze through the first wall's naţ ow opening and then rolled along its length to bypass the second larger wall with a relatively fast rolling speed. (B) The MMR jumped through a narrow slot, with frames captured in slow motion. (C) The MMR navigated through an unstructured terrestrial environment via the two-anchor and undulating crawling locomotion. The MMR was first commanded to two-anchor crawl across a path with strict shape constraints and subsequently climbed up a stair-like structure and moved cross a gap. To negotiate across a confined and enclosed channel with convoluted geometries, the 


\section{SI Video S6}

\section{Multimodal locomotion: Jumping and rolling}

Rich media available at https://drive.google.com/file/d/1yf91BYVsvHMY51NtFHmo0E1RPzfVJE9s/ view?usp=sharing

Next, we commanded the proposed MMR to overcome all the obstacles in the second unstructured terrain (Fig. 6C and SI Video S7). The MMR began by first rotating about its $y_{\{L\}}$-axis so that it could tilt and adopt a favorable orientation while executing the two-anchor crawling locomotion. This tilting was necessary as it allowed the actuator to two-anchor crawl across a path that had strict shape constraints (Fig. 6C and SI Video S7). Subsequently, we steered the MMR while it continued to use the two-anchor crawling locomotion to climb up the stair-like structure and move across the gap to reach the left side of the terrain (Fig. 6C and SI Video S7). The actuator was then commanded to negotiate across a confined and enclosed channel, which had convoluted geometries. To move across such a complex channel, the MMR activated its undulating crawling locomotion to ascend an upslope of $10^{\circ}$ until it reached a horizontal plane parallel to the ground (Fig. 6C and SI Video S7). Subsequently, it continued to use this locomotion in the horizontal plane to perform a sharp turn at the junction of an ' $\mathrm{L}$ '-shaped channel and transited to crawl across a vertical plane to reach its final destination (Fig. 6C and SI Video S7). We would like to highlight that existing soft MMRs would not be able to navigate across this unstructured terrain because they would not be able to tilt while performing the two-anchor crawling locomotion (Hu et al., 2018; Kim et al., 2011; Wu et al., 2020; Demir et al., 2021; Culha et al., 2020; Yang et al., 2020; Ju et al., 2021). As a result, they would not be able to cross the narrow path that had strict shape constraints. Furthermore, existing soft MMRs would not have the dexterity to undulating crawl across convoluted, enclosed channels with non-planar geometries (Hu et al., 2018; Ren et al., 2021; Du et al., 2020).

\section{SI Video $S 7$}

\section{crawling}

\section{Multimodal locomotion: Two-anchor crawling and undulating}

Rich media available at https://drive.google.com/file/d/179WATu4KafwUNRCuvyYwDQSa-cHOGAX8/ view?usp=sharing

The third unstructured environment, which the proposed MMR navigated in, was a hybrid aquatic-terrestrial environment (Fig. 6D and SI Video S8). The MMR began at the bed of the water reservoir, and it started to swim against gravity by executing the jellyfish-like swimming locomotion (Fig. 6D and SI Video S8, average speed: $9.37 \mathrm{~mm} \mathrm{~s}^{-1}$, Reynolds number: 67.2 ). Because the proposed MMR was able to precisely control its three axes of angular displacements, it was able to steer towards the opening of a barrier and subsequently adopted a favorable orientation which could allow it to negotiate across the opening (Fig. 6D and SI Video S8); otherwise, the MMR would not be able to cross this rigid obstacle. Upon reaching the water surface, the proposed MMR adopted a rolling locomotion to peel its surface off the air-water interface and eventually transited from water to land (Fig. 6D and SI Video S8). To allow the MMR to peel its surface off the air-water interface more easily, we had coated the buoyant components of the MMR with a thin layer of hydrophobic polymer (Ecoflex 00-10). Nonetheless, because the surface tension of the air-water interface was strong, the MMR had to perform several rounds of rolling before it could eventually break off this surface tension (SI Video S8). Another limitation of the MMR was that its free ends were found to be stuck to a 
water droplet after it landed. Hence, the MMR was unable to recover to its flat configuration and had to remain in the ' $U$ '-shaped configuration. In theory, this issue could be mitigated if the surfaces of the MMR could be coated with a thin layer of Teflon to become superhydrophobic because this would prevent the water droplets from sticking to the actuator after it emerged from water (Im et al., 2010; Van Der Wal \& Steiner, 2007). We aim to investigate this approach more thoroughly in the future. Despite these limitations, we would like to highlight that existing five-DOF, soft MMRs would not have the dexterity to overcome the obstacle in the reservoir because they could not fully control their orientation and adopt a favorable shape to swim through such a tight opening with strict shape constraints (Hu et al., 2018; Lum et al., 2016; Ren et al., 2019). Although the six-DOF, jellyfish-like MMR in our previous work could theoretically overcome this barrier too, it could not perform a similar rolling locomotion to transit from water to land (Xu et al., 2021). The experiments in Fig. 6 and SI Videos S6-8 therefore demonstrated that our proposed MMR had unprecedented dexterity since it was able to negotiate across difficult barriers that are impassable by existing small-scale devices (e.g., (Hu et al., 2018; Xu et al., 2021; Ren et al., 2021; Ren et al., 2019; Du et al., 2020)).

\section{SI Video $S 8$}

\section{Multimodal locomotion: Jellyfish-like swimming and landing}

Rich media available at https://drive.google.com/file/d/1ohZekOJdFFliUTitkPYryV231dgpUkD9/ view?usp=sharing

Because our proposed MMR had adequate stiffness, it could also perform 3D pick-and-place operations. For instance, our MMR could roll towards an object and control its 'U'-shaped curvature to grip that object (Fig. 6E(i)-(ii) and SI Video S9). Subsequently, the MMR could hold on to the object while transporting it to the desired placement location via the rolling locomotion (Fig. 6E(iii) and SI Video S9). Upon arrival, the MMR could reduce its gripping force and release the object (Fig. 6E(iv) and SI Video S9). By repeating this process for the second object (Fig. 6E(v)-(vii)), the MMR could successfully stack these objects on top of each other (Fig. 6E(viii) and SI Video S9). To evaluate the payload of our soft MMR, we had commanded our MMR to grab various objects and use the rolling locomotion to transport them to a desired placement location (Fig. S13). Our experiments indicated that the proposed MMR was able to carry objects that had 0.5 to 3 folds of its weight $(29.4 \mu \mathrm{N})$. We also observed that the MMR would not be able to grab an object firmly when the object's weight was increased to 3.5 times folds of its weight. In addition, we had computed the theoretical positioning resolution of our MMR during the pick-and-place operation. Assuming that the MMR was operating on an obstacle-free flat terrain, the angular resolution of the MMR when it was rotating about the $x_{\{L\}^{-}}$and $y_{\{L\}^{-a x e s}}$ would be $0.57^{\circ}$ (SI section S4F). The sixth-DOF angular resolution of the MMR would be $0.36^{\circ}$ (SI section S4F). These angular resolutions were computed based on the resolution of our actuating magnetic signals (SI section S4A). During the pick-and-place operation, the shape of the rolling MMR could be approximated as a circle, and the radius of this best fit circle was about $0.95 \mathrm{~mm}$ (Fig. S14). Under no-slip conditions, the theoretical translational resolution of the MMR when it rolled along its length could be computed as $9.5 \mu \mathrm{m}$ via the product of its radius and $y_{\{L\}}$-axis angular resolution (SI section $\mathrm{S} 4 \mathrm{~F})$. The positioning resolution, however, might become coarser if the no-slip condition was violated. In the future, we aimed to explore the feasibility of performing 3D pick-and-place operations in conjunction with the MMR's other modes of locomotion. As it was challenging to draw the magnetic actuating signals applied to our MMR in most of the figures and videos, we had instead presented these experimental signals separately in Fig.s S15-S31. 


\section{SI Video S9}

\section{D pick-and-place operation}

Rich media available at https://drive.google.com/file/d/12ERhoxl121qVGSd14zNdemUiNgfsi7nN/ view?usp=sharing

\section{Discussion}

In this section, we will provide additional discussions on the locomotion of our proposed MMR. Relevant comparisons between the proposed MMR and other similar existing devices will also be made here. To make our discussions more complete, the limitations of our MMR will also be explicitly discussed. Furthermore, we will also suggest feasible future works that may potentially address or mitigate these issues.

Similar to the five-DOF soft MMRs presented by $\mathrm{Hu}$ et al. (Hu et al., 2018), our proposed actuator can also perform undulating swimming and meniscus-climbing on an air-water interface (SI section S4E). Hence, the proposed MMR is able to execute a total of seven types of soft-bodied locomotion. However, possessing six-DOF will not enhance the dexterity of the MMR when it is executing the undulating swimming and meniscus-climbing gaits. This is because these two modes of locomotion require the MMRs to be constrained on an air-water interface (Hu et al., 2018). Therefore, the motions of the MMR are restricted to 2D for these gaits, and this has in turn eliminated the benefits of having the additional sixth-DOF rotation. In the future, we aim to endow the proposed MMR with more modes of soft-bodied locomotion, which can benefit from having six-DOF. We will also investigate feasible gaits that can eventually allow our proposed MMR to reliably transit from an air-liquid interface to the liquid bulk.

\section{SI Video S10}

\section{Undulating swimming locomotion}

Rich media available at https://drive.google.com/file/d/1s0ShtycGSgpD22xpXM4mzKi9Db-yT6oS/ view?usp=sharing

In theory, it is also possible to implement six-DOF control on previous soft MMRs with harmonic magnetization profiles (Hu et al., 2018; Ren et al., 2021; Diller et al., 2014; Zhang \& Diller, 2015; Zhang et al., 2016; Zhang \& Diller, 2018; Demir et al., 2021; Culha et al., 2020). The key reason why such MMRs can only achieve five-DOF motions previously is because their orientation is controlled solely by $\vec{B}$ (Hu et al., 2018 ; Ren et al., 2021; Diller et al., 2014; Zhang \& Diller, 2015; Zhang et al., 2016; Zhang \& Diller, 2018; Demir et al., 2021; Culha et al., 2020). Unlike these previous works, here we use both $\vec{B}$ and $\vec{B}_{\text {grad }}$ to control the orientation of the proposed MMR, and this enables us to generate a sixth-DOF restoring torque for this soft actuator, effectively allowing it to realize six-DOF motions. Although it is possible to implement six-DOF control on previous soft MMRs with harmonic magnetization profiles (Hu et al., 2018; Ren et al., 2021; Diller et al., 2014; Zhang \& Diller, 2015; Zhang et al., 2016; Zhang \& Diller, 2018; Demir et al., 2021; Culha et al., 2020), their producible sixth-DOF torque will be 1.41-63.9 folds lower than our proposed actuator.

While the proposed MMR had demonstrated unprecedented dexterity, it was controlled manually by a human operator in this first study. In the subsequent studies, we aim to increase the speed of the MMR by implementing closed-loop position control with visual feedback. For lab-on-chip applications, such visual feedback for the MMRs can be obtained via standard cameras (Chung et al., 2015). In the future, when MMRs are deployed for their targeted biomedical applications, ultrasound imaging technology would be a promising candidate for providing such visual feedback for these actuators (Hu et al., 2018). This is because 
recent studies have shown that the actuating magnetic fields for MMRs have minimal interference with ultrasound imaging systems (Hu et al., 2018). To make our proposed MMRs more favorable for potential medical applications, we aim to further scale down the size of these actuators in the future.

For this first study, our main objective is to investigate the enhancement in dexterity when a soft MMR is able to concurrently possess six-DOF motions and multimodal locomotion. Hence, we did not perform detailed characterization on the proposed MMR to evaluate the maximum speed achievable by each locomotion. We also did not evaluate the maximum height or range that the MMR could jump. Such in-depth experimental investigations can potentially be pursued in subsequent studies. In the future, we also aim to investigate if six-DOF control can be applied to soft MMRs with 3D magnetization profiles. This can potentially allow us to discover new MMRs that can realize higher sixth-DOF torque and yet capable of performing multimodal soft-bodied locomotion. The feasibility of optimizing the MMR's geometries via numerical methods (Lum et al., 2015; Lum et al., 2015; Lum et al., 2013; Lum et al., 2017) to further enhance its dexterity can similarly be explored in such further studies too.

While our proposed MMR can jump across complex barriers, it is difficult to accurately control the initial jumping velocity. This is because it is very challenging to model the dynamic interaction between the MMR and the substrate when the former strikes the latter to gain the necessary momentum to jump (Ng et al., 2021; Hu et al., 2018). In the future, we aim to investigate whether deep learning techniques can be employed to overcome this limitation (Silver et al., 2017). By obtaining experimental data to train the weights of the neural networks, it may be possible to develop numerical models that can accurately predict the MMR's initial jumping velocity. Using such numerical models, we can potentially allow the jumping locomotion to be more deterministic.

In summary, this work proposes a six-DOF MMR that can execute seven modes of soft-bodied locomotion and perform 3D pick-and-place operations. Based on the proposed control strategy, our MMR has demonstrated unprecedented dexterity by negotiating across barriers impassable by existing small-scale devices. We envision that this work can potentially inspire future MMRs to be significantly more dexterous, and this would be a critical step towards making these actuators practical for their targeted biomedical applications.

\section{Acknowledgments}

G.Z.L. is funded by the start-up grant awarded by Nanyang Technological University. The authors would like to thank C. S. X. Ng and N. Y. W. Foo from Nanyang Technological University for their help in revising the paper.

\section{Conflict of Interest}

The authors declare no conflict of interest.

\section{References}

Microrobots for minimally invasive medicine. (2010). Annual Review of Biomedical Engineering, 12, 55-85.

Soft actuators for small-scale robotics. (2017). Advanced Materials, 29.

Miniature soft robots - road to the clinic. (2018). Nature Reviews Materials, 3(6), 74-75. 
Electronically integrated, mass-manufactured, microscopic robots. (2020). Nature, 584, 557-561.

Biomedical applications of untethered mobile milli/microrobots. (2015). Proceedings of the IEEE, 103, 205-224.

Locomotion of miniature soft robots. (2021). Advanced Materials, 33, e2003558.

Micro/nanorobots for biomedicine: delivery, surgery, sensing, and detoxification. (2017). Science Robotics, 2.

Structured light enables biomimetic swimming and versatile locomotion of photoresponsive soft microrobots. (2016). Nature Materials, 15, 647-653.

Magnetically actuated microrobots as a platform for stem cell transplantation. (2019). Science Robotics, 4.

Small-scale soft-bodied robot with multimodal locomotion. (2018). Nature, 554, 81-85.

Biopsy with thermally-responsive untethered microtools. (2013). Advanced Materials, 25, 514-519.

Magnetic helical micromachines: fabrication, controlled swimming, and cargo transport. (2012). Advanced Materials, 24, 811-816.

Magneto-aerotactic bacteria deliver drug-containing nanoliposomes to tumour hypoxic regions. (2016). Nature Nanotechnology, 11, 941-947.

Multicompartment tubular micromotors toward enhanced localized active delivery. (2020). Advanced Materials, 32, e2000091.

Multifunctional surface microrollers for targeted cargo delivery in physiological blood flow. (2020). Science Robotics, 5 .

A swarm of slippery micropropellers penetrates the vitreous body of the eye. (2018). Science Advances, 4, eaat4388.

Trends in micro-/nanorobotics: materials development, actuation, localization, and system integration for biomedical applications. (2021). Advanced Materials, 33, e2002047.

Ultrasound Doppler-guided real-time navigation of a magnetic microswarm for active endovascular delivery. (2021). Science Advances, 7.

Mobile microrobots for bioengineering applications. (2017). Lab on a Chip, 17, 1705-1724.

Three-dimensional programmable assembly by untethered magnetic robotic micro-grippers. (2014). Advanced Functional Materials, 24(28), 4397-4404.

Magnetic soft micromachines made of linked microactuator networks. (2021). Science Advances, $\%$.

Three-dimensional heterogeneous assembly of coded microgels using an untethered mobile microgripper. (2015). Lab on a Chip, 15, 1667-1676.

Untethered micro-robotic coding of three-dimensional material composition. (2014). Nature Communications, 5, 3124 .

Liquid crystal elastomer-based magnetic composite films for reconfigurable shape-morphing soft miniature machines. (2021). Advanced Materials, 33, e2006191.

Bioinspired microrobots. (2018). Nature Reviews Materials, 3(6), 113-124.

Fabrication and characterization of magnetic microrobots for three-dimensional cell culture and targeted transportation. (2013). Advanced Materials, 25, 5863-5868.

A light-driven artificial flytrap. (2017). Nature Communications, 8, 15546. 
Bioinspired cilia arrays with programmable nonreciprocal motion and metachronal coordination. (2020). Science Advances, 6 .

Magnetic cilia carpets with programmable metachronal waves. (2020). Nature Communications, 11, 2637.

Shape-programmable magnetic soft matter. (2016). Proceedings of the National Academy of Sciences, 113, E6007-E6015.

Materials, actuators, and sensors for soft bioinspired robots. (2021). Advanced Materials, 33, e2003139.

Controlled flight of a microrobot powered by soft artificial muscles. (2019). Nature, 575, 324-329.

Hydrogel walkers with electro-driven motility for cargo transport. (2015). Scientific Reports, 5, 13622.

Bipolar conducting polymer crawlers based on triple symmetry breaking. (2018). Advanced Functional Materials, 28(25), 1705825.

Micrometer-sized electrically programmable shape-memory actuators for low-power microrobotics. (2021). Science Robotics, 6 .

Microrobotic tentacles with spiral bending capability based on shape-engineered elastomeric microtubes. (2015). Scientific Reports, 5, 10768.

Soft microrobots employing nonequilibrium actuation via plasmonic heating. (2017). Advanced Materials, 29.

Light-driven, caterpillar-inspired miniature inching robot. (2018). Macromolecular Rapid Communications, 39.

Shape-programmable liquid crystal elastomer structures with arbitrary three-dimensional director fields and geometries. (2021). Nature Communications, 12, 5936.

First jump of microgel; actuation speed enhancement by elastic instability. (2010). Soft Matter, 6(18), $4342-4345$.

Self-walking gel. (2007). Advanced Materials, 19(21), 3480-3484.

OctoMag: an electromagnetic system for 5-DOF wireless micromanipulation. (2010). IEEE Transactions on Robotics, 26(6), 1006-1017.

Small-scale magnetic actuators with optimal six degrees-of-freedom. (2021). Advanced Materials, 33, e2100170.

Printing ferromagnetic domains for untethered fast-transforming soft materials. (2018). Nature, 558, 274-279.

Millimeter-scale flexible robots with programmable three-dimensional magnetization and motions. (2019). Science Robotics, 4.

3D printed personalized magnetic micromachines from patient blood-derived biomaterials. (2021). Science Advances, 7, eabh0273.

Three-dimensional independent control of multiple magnetic microrobots via inter-agent forces. (2020). The International Journal of Robotics Research, 39(12), 1377-1396.

Magnetic dynamic polymers for modular assembling and reconfigurable morphing architectures. (2021). Advanced Materials, 33, e2102113.

Programming magnetic anisotropy in polymeric microactuators. (2011). Nature Materials, 10, 747-752.

Permanent magnet array-driven navigation of wireless millirobots inside soft tissues. (2021). Science Advances, 7, eabi8932. 
Dexterous magnetic manipulation of conductive non-magnetic objects. (2021). Nature, 598, 439-443.

Pros and cons: magnetic versus optical microrobots. (2020). Advanced Materials, 32, e1906766.

Soft-bodied adaptive multimodal locomotion strategies in fluid-filled confined spaces. (2021). Science Advances, $\%$.

Six-degree-of-freedom magnetic actuation for wireless microrobotics. (2016). The International Journal of Robotics Research, 35(1-3), 114-128.

Nanomagnetic encoding of shape-morphing micromachines. (2019). Nature, 575, 164-168.

Reprogrammable shape morphing of magnetic soft machines. (2020). Science Advances, 6 .

Voxelated three-dimensional miniature magnetic soft machines via multimaterial heterogeneous assembly. (2021). Science Robotics, 6.

Rapidly deployable and morphable 3D mesostructures with applications in multimodal biomedical devices. (2021). Proceedings of the National Academy of Sciences, 118.

Bioinspired soft microrobots with precise magneto-collective control for microvascular thrombolysis. (2020). Advanced Materials, 32, e2000366.

Programmable design and performance of modular magnetic microswimmers. (2021). Advanced Materials, 33, e2006237.

A magnetically guided self-rolled microrobot for targeted drug delivery, real-time X-Ray imaging, and microrobot retrieval. (2021). Advanced Healthcare Materials, 10, e2001681.

Robotic metamorphosis by origami exoskeletons. (2017). Science Robotics, 2.

Continuously distributed magnetization profile for millimeter-scale elastomeric undulatory swimming. (2014). Applied Physics Letters, 104 (17), 174101.

Multi-functional soft-bodied jellyfish-like swimming. (2019). Nature Communications, 10, 2703.

Effect of body stiffness distribution on larval fish-like efficient undulatory swimming. (2021). Science Advances, 7 .

Millimeter-scale magnetic swimmers using elastomeric undulations. (2015). 2015 IEEE/RSJ International Conference on Intelligent Robots and Systems (IROS), 1706-1711.

Independent control of two millimeter-scale soft-bodied magnetic robotic swimmers. (2016). 2016 IEEE International Conference on Robotics and Automation (ICRA), 1933-1938.

A magnetically controlled soft miniature robotic fish with a flexible skeleton inspired by zebrafish. (2021). Bioinspiration $\&$ Biomimetics, 16.

Shape-programmable magnetic miniature robots: a critical review In: Y. Sun, X. Wang, J. Yu (Eds) Fielddriven micro and nanorobots for biology and medicine (pp. 211-242). (2022). (pp. ). Springer.

Magnetic shape memory polymers with integrated multifunctional shape manipulation. (2020). Advanced Materials, 32, e1906657.

Untethered soft robots for future planetary explorations?. (2021). Advanced Intelligent Systems. https: //doi.org/10.1002/aisy.202100106

Reconfiguration, camouflage, and color-shifting for bioinspired adaptive hydrogel-based millirobots. (2020). Advanced Functional Materials, 30(10), 1909202.

Evolutionary algorithm-guided voxel-encoding printing of functional hard-magnetic soft active materials. (2020). Advanced Intelligent Systems, 2(8), 2000060. 
Reconfigurable multifunctional ferrofluid droplet robots. (2020). Proceedings of the National Academy of Sciences, 117, 27916-27926.

Ferrofluid droplets as liquid microrobots with multiple deformabilities. (2020). Advanced Functional Materials, 30(24), 2000138.

Reconfigurable ferromagnetic liquid droplets. (2019). Science, 365, 264-267.

A shapeshifting ferrofluidic robot. (2021). Soft Robotics, 8, 687-698.

Untethered miniature soft robots: modeling and design of a millimeter-scale swimming magnetic sheet. (2018). Soft Robotics, 5(6), 761-776.

Task space adaptation via the learning of gait controllers of magnetic soft millirobots. (2021). The International Journal of Robotics Research. https://doi.org/10.1177/02783649211021869

Learning of sub-optimal gait controllers for magnetic walking soft millirobots. (2020). Robotics Science and Systems (RSS). https://doi.org/10.15607/RSS.2020.XVI.070

Six-degrees-of-freedom remote actuation of magnetic microrobots. (2014). Robotics: Science and Systems (RSS). https://doi.org/10.15607/RSS.2014.X.013

An agglutinate magnetic spray transforms inanimate objects into millirobots for biomedical applications. (2020). Science Robotics, 5.

Dual-axial motion control of a magnetic levitation system using Hall-effect sensors. (2015). IEEE/ASME Transactions on Mechatronics, 21(2), 1129-1139.

Reconfigurable magnetic soft robots with multimodal locomotion. (2021). Nano Energy, 87, 106169.

A jumping soft robot driven by magnetic field. (2021). International Conference on Intelligent Robotics and Applications, 267-274.

An untethered crawling and jumping micro-robot. (2021). 21st International Conference on Solid-State Sensors, Actuators and Microsystems (Transducers) (IEEE), 353-356.

A robust superhydrophobic and superoleophobic surface with inverse-trapezoidal microstructures on a large transparent flexible substrate. (2010). Soft Matter, 6(7), 1401-1404.

Super-hydrophobic surfaces made from Teflon. (2007). Soft Matter, 3, 426-429.

Structural optimization for flexure-based parallel mechanisms-towards achieving optimal dynamic and stiffness properties. (2015). Precision Engineering, 42, 195-207.

Integrating mechanism synthesis and topological optimization technique for stiffness-oriented design of a three degrees-of-freedom flexure-based parallel mechanism. (2015). Precision Engineering, 39, 125-133.

A hybrid topological and structural optimization method to design a 3-DOF planar motion compliant mechanism. (2013). IEEE/ASME International Conference on Advanced Intelligent Mechatronics, 247-254.

An XY z flexure mechanism with optimal stiffness properties. (2017). IEEE International Conference on Advanced Intelligent Mechatronics (AIM), 1103-1110.

Mastering the game of Go without human knowledge. (2017). Nature, 550, 354-359. 